\title{
The expression of a tubby-like protein from Malus domestica (MdTLP7) enhances abiotic stress tolerance in Arabidopsis
}

\author{
Jianing $\mathrm{Xu}^{\dagger}$, Shanshan Xing ${ }^{\dagger}$, Qinghua Sun, Chunyan Zhan, Xin Liu, Shizhong Zhang ${ }^{*}$ and Xiaoyun Wang*
}

\begin{abstract}
Background: Tubby-like proteins (TLPS), characterized by a signature tubby domain, are widespread in plants and animals. To date, only plant TLPs involved in multifarious stress responses and male gametophyte development have been identified. However, studies on the molecular functions of plant TLPs are largely unknown.

Results: In this investigation, the roles of a TLP from Malus domestica (MdTLP7) in response to abiotic stresses were characterized by expressing it in Arabidopsis. The expression of wild-type full-length MdTLP7 (FL) significantly increased the stress tolerance of Arabidopsis seedlings to osmotic, salt, cold and heat stress, while the expression of truncated MdTLP7 containing only the tubby domain (Tub) also showed some function. Located on a central a helix surrounded by 12 anti-parallel $\beta$ strands in the tubby domain, the $K_{190} / R_{192}$ site may be involved in fixation to the plasma membrane, as shown by 3D homology modelling with animal TLPs. This site might play a crucial role in anti-stress functions since site-directed mutagenesis of MdTLP7 reduced stress tolerance. Subcellular localization showed that MdTLP7 was mainly localized in the plasma membrane in plant cells, suggesting that it might participate in the transduction of stress signals.
\end{abstract}

Conclusions: The results of this study showed that MdTLP7 could improve abiotic stress tolerance not only in bacteria but also in plants. The $\mathrm{K}_{190} / \mathrm{R}_{192}$ residues in the tubby domain were not only the plasma membrane binding site of MdTLP7 but also played a key role in stress tolerance. These results may provide a basis for further exploring the mechanism of anti-stress functioning and downstream target genes of plant TLPS.

Keywords: Tubby-like protein, MdTLP7, Functional site, Stress tolerance, Subcellular localization

\section{Background}

The tubby-like proteins (TLPs) are widely distributed in the animal and plant kingdoms. There are 5 members of the TLP family in mice, 4 in Homo sapiens, 9 in apple and 11 in Arabidopsis [1-4]. In mammals, many cellular functions of TLPs are involved in vesicular trafficking, the mediation of insulin signalling, gene transcription, G-protein signalling, and ribosomal RNA synthesis [5-9]. Although several TLPs in plants have been identified, their roles are elusive. In Arabidopsis, AtTLP3 and AtTLP9 are involved in ABA-dependent signalling during germination [3].

\footnotetext{
* Correspondence: shizhong@sdau.edu.cn; xyunwang@sdau.edu.cn † Jianing Xu and Shanshan Xing contributed equally to this work. College of Life Science, State Key Laboratory of Crop Biology, Shandong Agricultural University, Shandong Taian 271018, People's Republic of China
}

Several plant TLPs are upregulated under abiotic and biotic stress [4, 10-13].

Our previous study showed that a TLP gene from Malus domestica (MdTLP7) was upregulated in the transcriptional profile of apple under cold stress [14]. Heterologous expression of MdTLP7 significantly increased the stress tolerance of $E$. coli cells against different abiotic stresses [15]. In contrast to animal TLPs, MdTLP7 contains a highly conserved F-box domain at its $\mathrm{N}$-terminus in addition to the characteristic tubby domain [15]. In eukaryotes, F-box proteins participate in diverse cellular processes such as the response to stress, signal transduction, and the development of floral organs, primarily as a component of the Skp1-cullin-F-box (SCF) complex in protein ubiquitination [16-20].

Subcellular localization of TLPs clarifies their functions. Many mammalian and plant TLPs are usually 
localized in the plasma membrane and nucleus [12, 21]. As to the mechanism of TLP binding to the plasma membrane, a phosphatidylinositol-4,5-bisphosphate $\left(\mathrm{PIP}_{2}\right)$ binding site involving two conserved residues in the tubby domain, lysine $_{330}\left(\mathrm{~K}_{330}\right)$ and arginine ${ }_{332}\left(\mathrm{R}_{332}\right)$, interacted directly with the membrane lipid of the plasma membrane, as demonstrated in two TLPs from mouse (TUB and TULP1) [22]. These two positively charged phosphate-coordinating residues $\left(K_{330}\right.$ and $\left.R_{332}\right)$ are highly conserved not only in the animal TLPs but also in quite a few plant TLPs. The corresponding plasma membrane binding site in the tubby domain of AtTLP3 $\left(\mathrm{K}_{187} / \mathrm{R}_{189}\right)$ also localizes AtTLP3 in the plasma membrane. Mutations of these conservative residues to other residues could disrupt the localization of AtTLP3 to the plasma membrane [23]. $\mathrm{PIP}_{2}$ affects cell signalling by binding target proteins or enzymes [24]. When plants suffer abiotic stress-inducing agents such as mannitol, $\mathrm{NaCl}$ and $\mathrm{H}_{2} \mathrm{O}_{2}$ treatments, AtTLP3 can trigger signals by detaching from the plasma membrane and moving through the cytosol to the nucleus to regulate the expression of genes [12, 23]. As bipartite transcription factors (TFs), mouse TLPs have DNA binding domains near their C-terminus and transcription modulation segments at their $\mathrm{N}$-terminus [5]. TFs usually bind to cis-regulatory regions of a gene, which act as molecular switches controlling various biological processes including abiotic and biotic stress responses [25, 26]. A chickpea TLP (CaTLP1) was demonstrated to be a putative TF presumably involved in multivariate stresses [11]. A rice TLP (OsTLP2) has been shown to bind to the promoter of OsWRKY13 to regulate disease resistance [10]. These reports suggest that TLPs might participate in stress signal transduction as TFs.

To determine the functions of MdTLP7 in plants, full-length and truncated MdTLP7 (without the F-box domain) were transformed into Arabidopsis in this study. The responses of transgenic plants to different stresses were assessed. Using 3D structure modelling and site-directed mutation experiments, we found a critical site in the tubby domain of MdTLP7 for abiotic stress tolerance. These results open up new horizons for studying the mechanism of action of stress tolerance in plant TLPs and may promote the development of methods to improve plant stress resistance.

\section{Results}

\section{Expression of MdTLP7 enhanced abiotic stress tolerance in Arabidopsis}

To examine the function of MdTLP7 in the plant stress response, the full-length cDNAs of wild-type full-length MdTLP7 (FL) and truncated MdTLP7 with only the tubby domain (Tub) were transformed into wild-type Arabidopsis (WT) by the CaMV 35S promoter. More than 10 transgenic lines were identified by kanamycin testing and PCR amplification (T1 generation, Additional file 1: Figure S1). Homozygous lines were identified by screening for non-segregation from each independent transformant (T3 generation). The 2 homozygous FL transgenic lines (named FL-1, FL-2) and 2 Tub transgenic lines (named Tub-1, Tub-2) with high expression were chosen for further analysis (Additional file 2: Figure S2).

Under PEG and salt treatments, the growth of both transgenic lines, FL and Tub, was better than that of WT; little difference was found between the FL and Tub lines (Fig. 1a and b). The results suggested that the expression of wild-type MdTLP7 (FL) or truncated MdTLP7 (Tub) enhanced tolerance to osmotic and salt stresses.

To induce temperature stress, we placed seedlings in hot $\left(45^{\circ} \mathrm{C}\right)$ and cold $\left(-20^{\circ} \mathrm{C}\right)$ environments. After treatment at $45^{\circ} \mathrm{C}$ for $12 \mathrm{~h}$, obvious differences were observed among the transgenic lines (FL and Tub) and between the transgenic lines and WT Arabidopsis, and the growth of the Tub plants was better than that of the WT plants but worse than that of the FL plants. After 3 days of recovery, all of the WT plants were dead whereas $80 \%$ of the FL transgenic plants and $40 \%$ of the Tub transgenic plants survived (Fig. 1c). Similar results after cold treatment were observed among the WT, FL and Tub lines. The seedlings were pre-treated at $5^{\circ} \mathrm{Cto}$ $-5^{\circ} \mathrm{C}$ for $10 \mathrm{~h}$ and then subjected to $-20^{\circ} \mathrm{C}$ for $40 \mathrm{~min}$. All of the WT plants were dead whereas $70 \%$ of the FL plants and $30 \%$ of the Tub plants survived after 3 days of recovery (Fig. 1d).

The results indicated that the expression of MdTLP7 in Arabidopsis could enhance tolerance to several abiotic stressors in Arabidopsis. Truncated MdTLP7 (Tub) still retained almost complete function compared to that of wild-type MdTLP7 (FL) under both PEG and salt stress. Regarding temperature stress, truncated MdTLP7 (Tub) only had approximately half or less of the anti-stress function of wild-type MdTLP7 (FL). The results suggested that the tubby domain of MdTLP7 has key roles and that the F-box domain may have synergistic effects with the tubby domain in the stress response.

\section{MdTLP7 is localized in the plasma membrane}

A 3D homology model of the tubby domains of MdTLP7 was established using SWISS-MODEL that is highly consistent with the mouse TLP (TULP3) structure. Both contain a central $\alpha$ helix and a closed 12-stranded $\beta$ barrel (Fig. 2a and b). Mouse TLPs are tethered to the plasma membrane via a $\mathrm{PIP}_{2}$ binding site $\left(K_{330} / R_{332}\right)$ in the tubby domain [8]. The corresponding amino acid residues in $M d \mathrm{TLP} 7, \mathrm{~K}_{190} / \mathrm{R}_{192}$, show high positional overlap with residues $K_{330} / R_{332}$ 


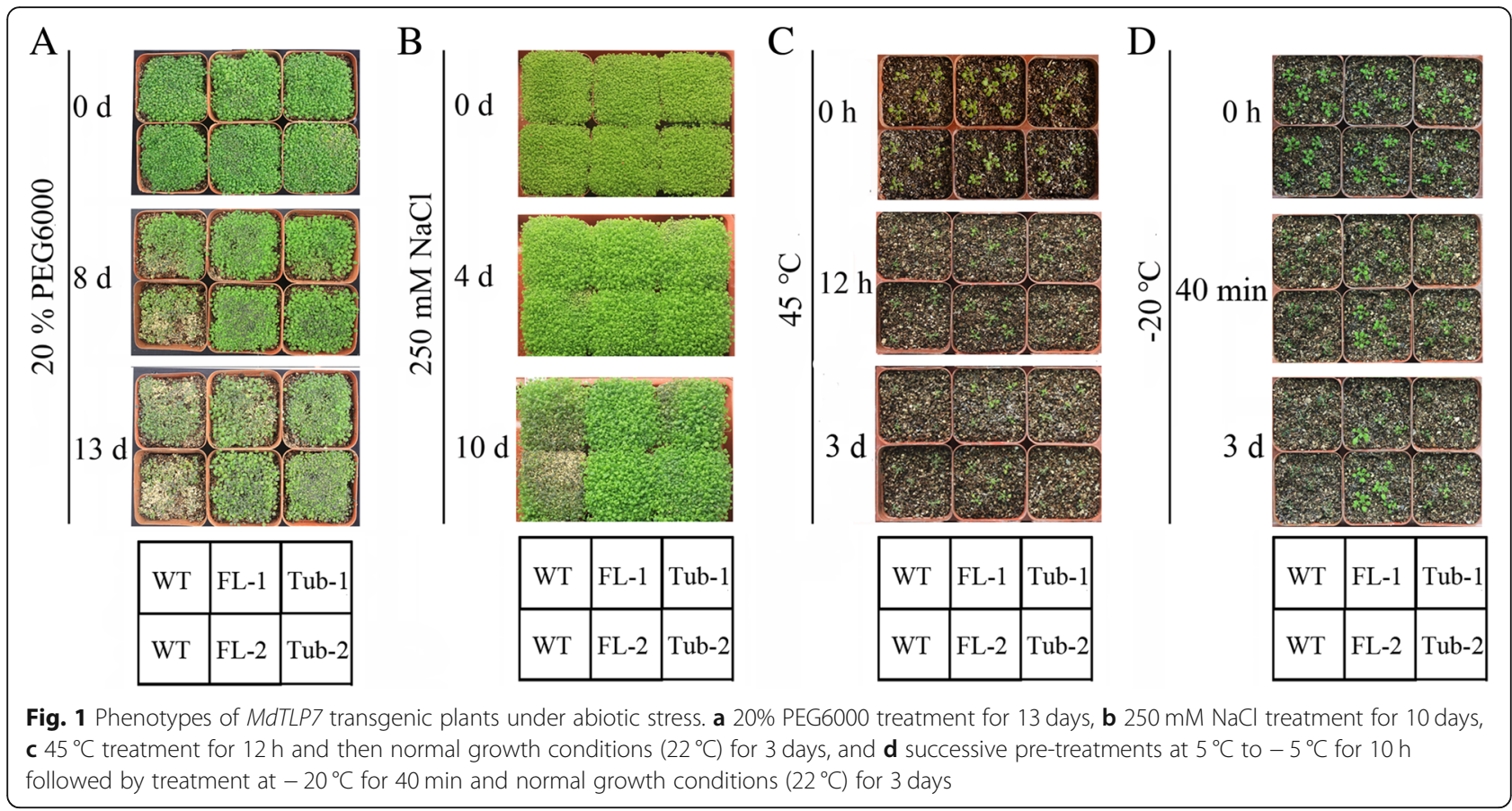

\section{MdTLP7}

A

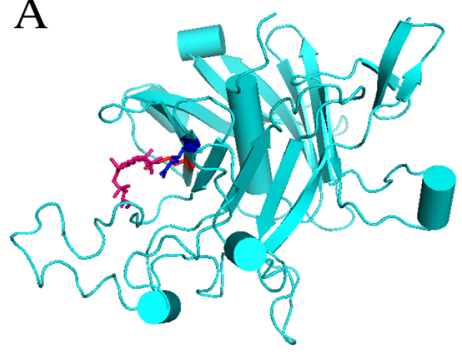

C

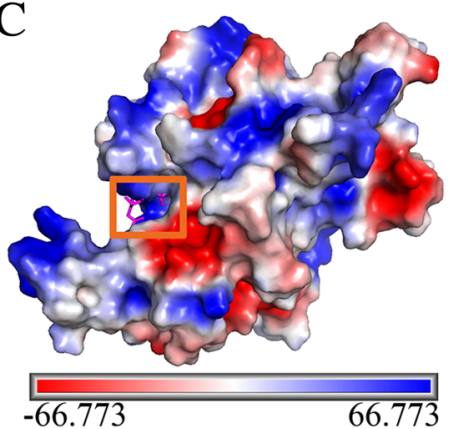

Mouse TLP

B

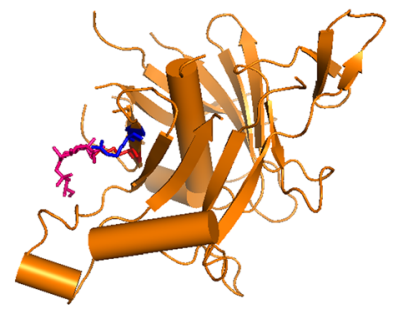

D

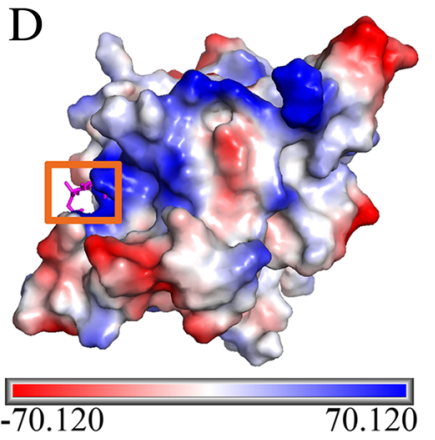

Fig. 2 3D structures of the tubby domain of MdTLP7 and mouse tubby protein. a-b 3D homology model of MdTLP7 (cyan) and mouse tubby protein (PDB identifier 117E; orange) showing $\mathrm{K}_{190}$ (red) and $\mathrm{R}_{192}$ (blue). IBS (pink), an analogue of PIP2, was used to show the conserved binding site. $\mathbf{c}-\mathbf{d}$ Electrostatic surface of the tubby domain generated with the programme PyMOL. Electrostatic surface of MdTLP7 with IBS modelled (c, orange frame) and mouse tubby protein-bound IBS (d, orange frame). The positive charges, negative charges and neutral charges are blue, red and white, respectively 
of mouse TLP, the TLP plasma membrane binding site. Both grooves of the plasma membrane binding site in MdTLP7 and mouse TLP were positively charged as shown by electrostatic surface analysis (Fig. 2c and d). The results suggested that MdTLP7 may also localize in the plasma membrane of plant cells, similar to mouse TLP in animal cells.

To investigate whether MdTLP7 localized in the plasma membrane, the sequence encoding $M d T L P 7$ was fused to the sequence of GFP. As shown in Fig. 3, the GFP signal emitted by the fusion protein was localized in the plasma membrane of tobacco leaf cells, while the GFP signal of the empty vector was localized in the cytoplasm and plasma membrane.

\section{$\mathrm{K}_{190} / \mathrm{R}_{192}$ in the tubby domain played crucial roles in} stress tolerance

A $\mathrm{PIP}_{2}$ analogue, L- $\alpha$-glycerophospho-D-myoinositol4,5-bisphosphate (IBS), was used to check changes in the charge of the corresponding plasma membrane binding site of $M d T L P 7$. When $\mathrm{K}_{190} / \mathrm{R}_{192}$ were mutated to alanine residues, the electrostatic surface of the IBS-bound groove changed from positive to neutral charge, although the whole tertiary structure of the tubby domain was not significantly affected in 3D homology modelling (Additional file 3: Figure S3 and Additional file 4: Figure S4). Whether the positive charge of the active groove is indispensable for the function of MdTLP7 needs to be identified.

To investigate the effects of this site on the anti-stress response, point mutants of $M d \mathrm{TLP}\left(\mathrm{K}_{190} \mathrm{~A}, \mathrm{R}_{192} \mathrm{~A}\right.$ or $\mathrm{K}_{190} \mathrm{~A} / \mathrm{R}_{192} \mathrm{~A}$ ) were constructed and transformed into $E$. coli. A survival test on solid medium showed that the survival ratio of cells expressing wild-type MdTLP7 was significantly higher than that of MdTLP mutants under salt stress conditions (Additional file 5: Figure S5).
The growth curves of wild-type MdTLP7 and MdTLP7 mutants in $500 \mathrm{mM} \mathrm{NaCl}$-containing liquid medium were assayed to further ascertain the site conferring stress resistance to MdTLP7. The cells expressing wild-type MdTLP7 entered the exponential growth stage after $10 \mathrm{~h}$ of cultivation, which was much earlier than the cells expressing mutant MdTLP7 $\left(\mathrm{K}_{190} \mathrm{~A}, \mathrm{R}_{192} \mathrm{~A}\right.$ or $K_{190} \mathrm{~A} / \mathrm{R}_{192} \mathrm{~A}$ ). The three mutants entered the exponential growth stage at approximately $15 \mathrm{~h}$, which was quite similar to the time frame of the empty vector (Table 1 and Fig. 4). These results revealed that the plasma membrane biding site at $\mathrm{K}_{190} / \mathrm{R}_{192}$ of $M d \mathrm{TLP7}$ played a key role in abiotic stress tolerance.

\section{Discussion}

The tubby domain is the signature conserved domain of the tubby-like family [9]. In addition to the tubby domain, almost all of the plant TLPs have an F-box domain at their N-terminal regions [21]. MdTLP7 has two functional domains: an F-box domain at the $\mathrm{N}$-terminus and a tubby domain at the C-terminus [4]. Some F-box domain-containing proteins are involved in the stress response by interacting with S-phase kinase association protein 1 to form an SCF complex and participating in the ubiquitin-proteasome system [27]. In our previous study, the F-box domain of MdTLP7 was not essential for the anti-stress function of bacteria since truncation of the F-box did not significantly affect its anti-stress resistance [15]. In this study, only expressing the Tub domain of MdTLP7 in Arabidopsis significantly increased tolerance to salt and PEG stress. Regarding cold and heat stress, the expression of full-length MdTLP7 was much better for tolerating cold and heat stress than the expression of truncated MdTLP7. The presence of the conserved F-box domain in MdTLP7 might play a role in stress tolerance through the ubiquitin-proteasome

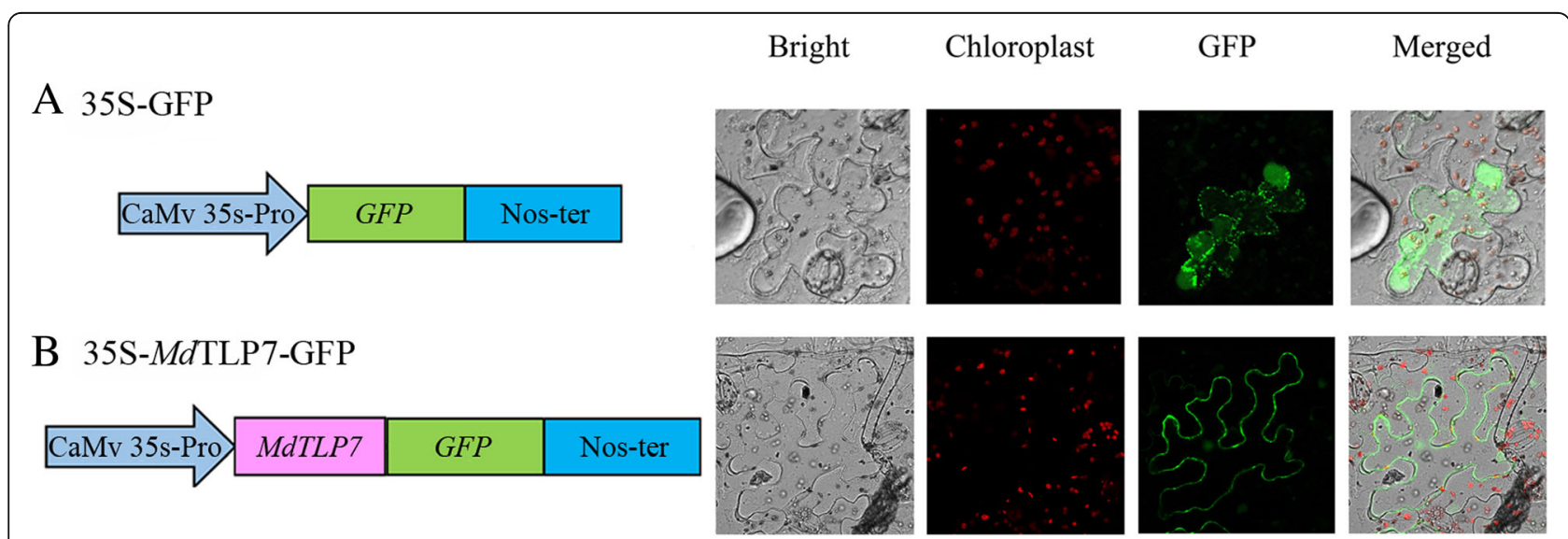

Fig. 3 MdTLP7 was localized in the PM of tobacco cells by the PIP 2 binding site. a The subcellular location of 35S-GFP protein. $\mathbf{b}$ The subcellular location of 35S-MdTLP7-GFP protein. Green fluorescence indicates the location of GFP. Red fluorescence indicates the location of the chloroplast 
Table 1 Nonlinear regression analysis of E. coli cell lines growth curve under salt stress

\begin{tabular}{llll}
\hline Sample & Equation & $r^{2}$ & Inflection point of time $(\mathrm{h})$ \\
\hline Empty vector & $\mathrm{Y}=1.830 /\left(1+1066 \mathrm{e}^{-0.458 \mathrm{t}}\right)$ & 0.987 & 15.2 \\
MdTLP7 & $\mathrm{Y}=1.703 /\left(1+7956 \mathrm{e}^{-0.837 \mathrm{t}}\right)$ & 0.995 & 10.7 \\
$\mathrm{~K}_{190} \mathrm{~A}$ & $\mathrm{Y}=1.700 /\left(1+2111 \mathrm{e}^{-0.539 \mathrm{t}}\right)$ & 0.970 & 14.2 \\
$\mathrm{R}_{192} \mathrm{~A}$ & $\mathrm{Y}=1.834 /\left(1+13032 \mathrm{e}^{-0.598 \mathrm{t}}\right)$ & 0.994 & 15.8 \\
$\mathrm{~K}_{190} \mathrm{~A} / \mathrm{R}_{192} \mathrm{~A}$ & $\mathrm{Y}=1.783 /\left(1+6736 \mathrm{e}^{-0.602 \mathrm{t}}\right)$ & 0.991 & 14.6 \\
\hline
\end{tabular}

pathway, which is a major posttranscriptional regulatory process related to redundant proteins in eukaryotes.

Boggon et al. determined that mouse TLPs were localized in the plasma membrane via a plasma membrane binding site $\left(K_{330} / R_{332}\right)$ in a structure of the tubby domain bound to $\mathrm{PIP}_{2}$ [5]. The 3D structure showed high positional overlap between $\mathrm{K}_{190} / \mathrm{R}_{192}$ in $M d \mathrm{TLP} 7$ and $\mathrm{K}_{330} /$ $\mathrm{R}_{332}$ in mouse TLP (Fig. 2). Therefore, MdTLP7 might also localize in the plasma membrane via $K_{190} / R_{192}$ binding to $\mathrm{PIP}_{2}$. $\mathrm{PIP}_{2}$ is a kind of membrane phospholipid that affects cell signalling by binding target proteins to generate bioactive inositol phosphates [24]. In this investigation, we found that MdTLP7 localized in the plasma membrane of tobacco cells, suggesting that it might participate in stress signal transduction (Fig. 3). However, studies on the relationship between the plasma membrane binding site and the protein functions of plant TLP have not been reported. The survival ratios of cells expressing MdTLP7 point mutants $\left(\mathrm{K}_{190} \mathrm{~A}\right.$ and $\left.\mathrm{R}_{192} \mathrm{~A}\right)$ were slightly higher than that of cells expressing the empty vector under stress. However, the survival ratio of cells expressing MdTLP7 was significantly higher than that of cells expressing MdTLP7 mutants under salt stress condition (Additional file 5: Figure S5). The growth curve assay also showed that the cells expressing wild-type MdTLP7 entered the exponential growth stage much earlier than the cells expressing mutant MdTLP7 (Table 1 and Fig. 4). The survival tests on solid and liquid medium both revealed that the plasma membrane binding site at $K_{190} / R_{192}$ of MdTLP7 played a key role in abiotic stress tolerance. The 3D homology model and electrostatic surface analysis of $M d$ TLP7 showed that point mutations at $\mathrm{K}_{190} / \mathrm{R}_{192} \mathrm{did}$ not change the 3D structure of $M d T L P 7$, but the $\mathrm{PIP}_{2}$ binding groove changed from positively charged to neutral, indicating that the positive charge of the groove was important to the anti-stress function of MdTLP7 (Additional file 3: Figure S3 and Additional file 4: Figure S4).

\section{Conclusions}

The results of this study showed that MdTLP7 could improve abiotic stress tolerance not only in bacteria but also in Arabidopsis. The expression of full-length wild-type MdTLP7 significantly increased the stress tolerance of Arabidopsis seedlings to osmotic, salt, cold and heat stress, while the expression of truncated MdTLP7 including only the tubby domain also retained some function. The $K_{190} / R_{192}$ residues of $M d T L P 7$ were found to be key amino acids involved in stress tolerance for the first time. In the future, we will further study the molecular mechanism of MdTLP7 in abiotic stress tolerance.

\section{Methods}

Expression of MdTLP7 in transgenic plants

In this study, Arabidopsis thaliana ecotype Columbia-0 (Col-0) was used as the experimental material. MdTLP7 cDNA containing the complete open reading frame

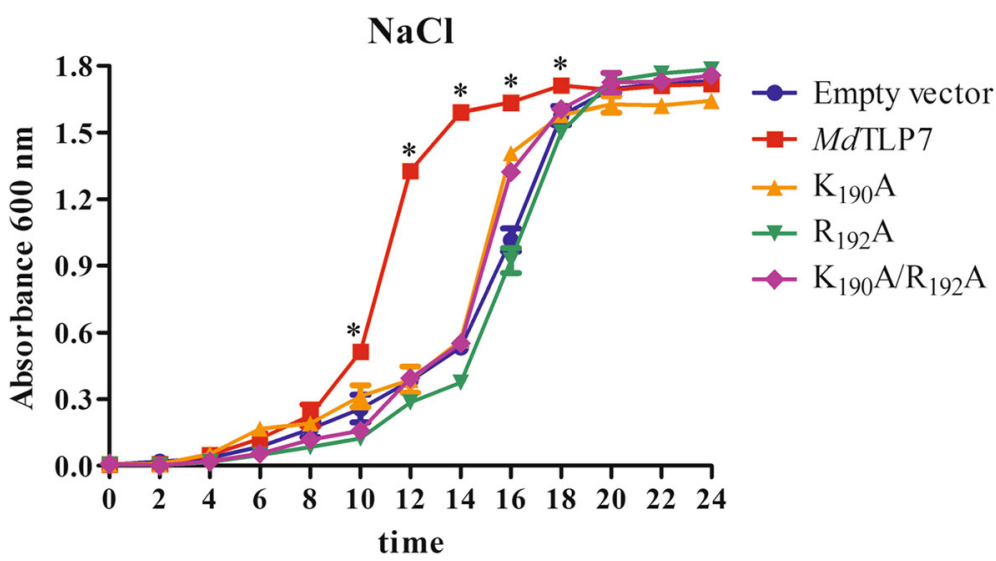

Fig. 4 Growth analysis of E. coli cells expressing full length MdTLP7 and three MdTLP7 point mutants. E. coli cells were cultivated in LB medium supplemented with $500 \mathrm{mM} \mathrm{NaCl}$. OD600 was recorded at $2 \mathrm{~h}$ intervals for $24 \mathrm{~h}$, and the mean values are represented in the graph. ${ }^{*} p<0.001$ 
(ORF) and cDNA containing the tubby domain of MdTLP7 were amplified from the MdTLP7-pET30a recombinant vector, which was constructed in our previous study [15]. The full-length MdTLP7 (FL) and tubby domain (Tub) cDNAs were inserted into the pBI121 vector, resulting in two recombinant vectors (pBI121-FL, pBI121-Tub). The pBI121-FL and pBI121-Tub recombinant vectors were integrated into Arabidopsis Columbia-0 by Agrobacterium tumefaciens (GV3101)-mediated transformation. The floral dip method was used for this transformation [28]. Transformants (T1) were selected on Murashige and Skoog medium agar plates $(50 \mu \mathrm{g} / \mathrm{mL}$ kanamycin). The presence and integrity of the FL or Tub cDNAs in transformants (T1) were further confirmed by PCR amplification. The primer sequences used in this experiment are listed in Additional file 6: Table S1. The seeds from the putative transgenic plants were again selfed to obtain $\mathrm{T} 3$ progenies through $\mathrm{T} 2$ plants. The transgenic (T3 generation) and WT Arabidopsis seeds were used for further experiments.

Quantitative real-time PCR (qRT-PCR) was performed to investigate the expression of FL and Tub in Arabidopsis. Two FL transgenic plants (named FL-1 and FL-2), Tub transgenic plants (named Tub-1 and Tub-2) and wild-type (WT) Arabidopsis plants were randomly selected. FL-1 was used as a control, and the actin gene was used as an internal control. The primer sequences used in this experiment are listed in Additional file 6: Table S1. The experiments were carried out at least three times under identical conditions. The relative expression levels were calculated using the $2^{-(\Delta \Delta C t)}$ method [29].

\section{Plant growth conditions and stress treatments}

The WT, FL and Tub plants were grown in vermiculite at a constant temperature of $22^{\circ} \mathrm{C}$ under $8 \mathrm{~h}$ illumination at $120 \mu \mathrm{mol} \mathrm{m}^{-2} \mathrm{~s}^{-1}$ and a $16 \mathrm{~h}$ dark cycle. WT, FL and Tub plants were grown to 10 days of age. To induce osmotic stress, 10-day-old seedlings were irrigated with a $20 \%$ PEG6000 solution every day for up to 13 days. To induce salt stress, 10-day-old seedlings were irrigated with a 250 $\mathrm{mM} \mathrm{NaCl}$ solution every day for up to 10 days.

Forty Arabidopsis plants from four independent lines grown for 10 days at $22^{\circ} \mathrm{C}$ were subjected to temperature stress treatment. To induce heat stress, 10-day-old seedlings were grown at $45^{\circ} \mathrm{C}$ for $12 \mathrm{~h}$ and then returned to a growth chamber at $22^{\circ} \mathrm{C}$ under $8 \mathrm{~h}$ illumination at $120 \mu \mathrm{mol} \mathrm{m}^{-2} \mathrm{~s}^{-1}$ and a $16 \mathrm{~h}$ dark cycle for 3 days. To induce cold stress, 10-day-old seedlings were pre-treated at $5{ }^{\circ} \mathrm{C}$ to $-5^{\circ} \mathrm{C}$ for $10 \mathrm{~h}$ successively and then subjected to $-20^{\circ} \mathrm{C}$ for $40 \mathrm{~min}$. After cold treatment, the seedlings were returned to a growth chamber at $22^{\circ} \mathrm{C}$ under $8 \mathrm{~h}$ illumination at $120 \mu \mathrm{mol} \mathrm{m}^{-2} \mathrm{~s}^{-1}$ and a $16 \mathrm{~h}$ dark cycle for 3 days before scoring the survival of the seedlings.

\section{Subcellular localization of MdTLP7}

To determine the subcellular localization of MdTLP7, the MdTLP7 ORF without a termination codon was inserted upstream of the GFP gene. The 35S-GFP plasmid was used as a control. The subcellular localization experiment was carried out by Agrobacterium tumefaciens infiltration into the leaves of tobacco as described by Jia et al. [30]. After $48 \mathrm{~h}$ of infiltration, a two photon laser confocal microscope (ZEISS, Germany) was used to observe the fluorescence in tobacco cells. Fluorescence was detected using a 505 to $550 \mathrm{~nm}$ bandpass filter for GFP. Image processing was performed with the Zeiss LSM image processing software (Zeiss).

\section{Construction of MdTLP7 site-directed mutants}

Three point mutations $\left(\mathrm{K}_{190} \mathrm{~A}, \mathrm{R}_{192} \mathrm{~A}\right.$ and $\left.\mathrm{K}_{190} \mathrm{~A} / \mathrm{R}_{192} \mathrm{~A}\right)$ were made by a QuikChange ${ }^{\text {Tx }}$ mutagenesis kit using the mutant primers listed in Additional file 6: Table S1. The three MdTLP7 point mutants were all inserted into the pET30a $(+)$ expression plasmid (Novagen) and transformed into E. coli cells. All the constructed plasmids were sequenced by Sunny Biotechnology Company (Shanghai China) to confirm the correct sequences.

\section{Survival test under salt stress on solid medium and in solution medium}

The survival test and growth analysis under salt stress of the E. coli described above were carried out as described by $\mathrm{Du}$ et al. [15]. The cells were grown in LB solution medium to an OD600 of $0.4-0.6$ at $37^{\circ} \mathrm{C}$, and expression of the recombinant proteins was induced for $2 \mathrm{~h}$ with 0.5 $\mathrm{mM}$ isopropyl- $\beta$-D-thiogalactopyranoside at $37^{\circ} \mathrm{C}$. For the survival test on solid medium, cultures were diluted to an OD600 of 0.6 and then diluted to $10^{-2}, 10^{-3}$ and $10^{-4}$. Ten microliter cultures from each dilution $\left(10^{-2}, 10^{-3}\right.$ and $10^{-4}$ ) were plated on solid LB medium supplemented with $500 \mathrm{mM} \mathrm{NaCl}$ and then incubated at $37^{\circ} \mathrm{C}$ for $16 \mathrm{~h}$. The colony number on each plate for the culture diluted to $10^{-3}$ was counted after incubation. Each experiment was repeated 3 times, and the data are presented as the mean with error bars indicating the standard deviation. The significance of the differences between groups was estimated using Student's $t$-test. A value of $P<0.01$ indicated a significant difference.

For the survival test in solution medium, cultures were diluted to an OD600 of 0.6 and $20 \mu \mathrm{l}$ of the cultures were used to inoculate $10 \mathrm{ml} \mathrm{LB}$ solution containing a high concentration of $500 \mathrm{mM} \mathrm{NaCl}$ before incubation at $37^{\circ} \mathrm{C}$ on a rotary shaker $(150 \mathrm{rpm})$. The bacterial suspension was harvested every $2 \mathrm{~h}$ until $24 \mathrm{~h}$ had passed, and the OD600 was measured. Each experiment was repeated 3 times, and the data are presented as the mean with error bars indicating the standard deviation. All statistical analyses were performed using SPSS 19.0 
(IBM, SPSS, Chicago, IL). Figures were made by GraphPad Prism 5 (GraphPad Software Inc., San Diego, CA, USA). Nonlinear regression analysis was fit using a logistic model $\left(\mathrm{Y}=\mathrm{A} /\left(1+\mathrm{Be}^{-\mathrm{kt}}\right)\right)$. The inflection point of the time $((\ln B) / k)$ of each sample was calculated. The significance of the differences between groups was estimated using Student's $t$-test. A value of $P<0.001$ indicated a significant difference.

\section{D structure modelling}

The 3D structural models of MdTLP7 and MdTLP7 mutants were constructed using the protein structure homology-modelling server SWISS-MODEL [31-33]. The most suitable template structure searched by the SWISS-MODEL server was used. To compare the spatial position of MdTLP7 bound to $\mathrm{PIP}_{2}$, a mouse brain tubby protein-bound $\mathrm{PIP}_{2}$ structure (PDB identifier 1I7E) was used as a 3D model. Visualization of the protein molecules and vacuum electrostatics analysis was performed using the PyMOL Molecular Graphics System (Version 1.7.4.5, https://pymol.org/2/, Schrödinger, LLC).

\section{Additional files}

Additional file 1: Figure S1. PCR amplification of Kan-resistant seedlings. (1-10) PCR products of $\mathrm{OE}_{\mathrm{FL}}$ transgenic lines, (11-20) PCR products of OE transgenic lines, M, DL2000 marker. (TIF 502 kb)

Additional file 2: Figure S2. qRT-PCR analysis of the expression of MdTLP7 in the leaves of WT and transgenic plants. (TIF $1457 \mathrm{~kb}$ )

Additional file 3: Figure S3. Homology model of the Tubby domain of MdTLP7 and three MdTLP7 point mutants. (A) Tubby domain of MdTLP7. (B-D) Three MdTLP7 point mutants. $K_{190}$ is shown in red, $R_{192}$ in blue, and mutated amino acids in orange. (TIF $1046 \mathrm{~kb}$ )

Additional file 4: Figure S4. Electrostatic surface of the tubby domain of MdTLP7 and three MdTLP7 point mutations. (A) Electrostatic surface of the MdTLP7 tubby protein. (B-D) Electrostatic surfaces of the point mutants. Blue indicates a positive charge, red indicates a negative charge and white indicates a neutral charge. The groove of the IBS-bound region is shown in the green frame. (TIF $2105 \mathrm{~kb}$ )

Additional file 5: Figure S5. Survival test of E. coli expressing MdTLP7, empty vector or point mutants of under salt stress. (A) $10 \mu \mathrm{l}$ cultures from $10^{-2}$ to $10^{-4}$ dilutions were spotted on LB plates treated with $0.5 \mathrm{M}$ $\mathrm{NaCl}$. (B) The colony numbers for the $10^{-3}$ dilutions appearing on each plate were counted. ${ }^{*} p<0.01$. (TIF $13567 \mathrm{~kb}$ )

Additional file 6: Table S1. Primers used in this experiment. (DOCX $12 \mathrm{~kb}$ )

\section{Abbreviations}

3D: three-dimensional; A: alanine; FL: full-length of MdTLP7; IBS: L-aglycerophospho-D-myoinositol 4,5-bisphosphate; K: lysine; ORF: open reading frame; PIP : phosphatidylinositol-4,5-bisphosphate; qRT-PCR: Quantitative realtime PCR; R: arginine; SCF: Skp1-cullin-F-box; TF: transcription factor; TLP: tubby-like protein; Tub: tubby domain; WT: wild-type of Arabidopsis

\section{Acknowledgements}

Not Applicable.

\section{Funding}

This work was supported by National Natural Science Foundation of China (31872042). The Funding bodies were not involved in the design of the study and collection, analysis, and interpretation of data and in writing the manuscript.

\section{Availability of data and materials}

The datasets used and/or analysed during the current study are available from the corresponding author on reasonable request.

\section{Authors' contributions}

Designed the experiments: XW, SX and JX. Performed the experiments: SX JX and CZ. Analyzed the data: XL, JX and SZ. Wrote the paper: XW, QS and JX. All authors have read and approved the final manuscript.

Ethics approval and consent to participate

Not applicable.

Consent for publication

Not applicable.

\section{Competing interests}

The authors declare that they have no competing interests.

\section{Publisher's Note}

Springer Nature remains neutral with regard to jurisdictional claims in published maps and institutional affiliations.

Received: 24 October 2018 Accepted: 24 January 2019

Published online: 06 February 2019

References

1. North MA, Naggert JK, Yan Y, Noben-Trauth K, Nishina PM. Molecular characterization of TUB, TULP1, and TULP2, members of the novel tubby gene family and their possible relation to ocular diseases. Proc Natl Acad Sci. 1997:94:3128-33.

2. Nishina PM, North MA, Ikeda A, Yan Y, Naggert JK. Molecular characterization of a novel tubby gene family member, TULP3, in mouse and humans. Genomics. 1998;54:215-20.

3. Lai CP, Lee CL, Chen PH, Wu SH, Yang CC, Shaw JF. Molecular analyses of the Arabidopsis TUBBY-like protein gene family. Plant Physiol. 2004;134: 1586-97.

4. Xu JN, Xing SS, Zhang ZR, Chen XS, Wang XY. Genome-wide identification and expression analysis of the tubby-like protein family in the Malus domestica genome. Front Plant Sci. 2016;7:1693

5. Boggon TJ, Shan WS, Santagata S, Myers SC, Shapiro L. Implication of tubby proteins as transcription factors by structure-based functional analysis. Science. 1999;286:2119-25.

6. Kapeller R, Moriarty A, Strauss A, Stubdal H, Theriault K, Siebert E, et al Tyrosine phosphorylation of tub and its association with Src homology 2 domain-containing proteins implicate tub in intracellular signaling by insulin. J Biol Chem. 1999;274:24980-6.

7. He W, Ikeda S, Bronson RT, Yan G, Nishina PM, North MA, Naggert JK. GFPtagged expression and immunohistochemical studies to determine the subcellular localization of the tubby gene family members. Mol Brain Res. 2000;81:109-17.

8. Santagata S, Boggon TJ, Baird CL, Gomez CA, Zhao J, Shan WS, Myszka DG, Shapiro L. G-protein signaling through tubby proteins. Science. 2001;292: 2041-50.

9. Ikeda A, Nishina PM, Naggert JK. The tubby-like proteins, a family with roles in neuronal development and function. J Cell Sci. 2002;115:9-14.

10. Cai M, Qiu D, Yuan T, Ding X, Li H, Duan L, Xu C, Li X, Wang S. Identification of novel pathogen-responsive cis-elements and their binding proteins in the promoter of OsWRKY13, a gene regulating rice disease resistance. Plant Cell Environ. 2008;31:86-96.

11. Wardhan V, Jahan K, Gupta S, Chennareddy S, Datta A, Chakraborty S, Chakraborty N. Overexpression of CaTLP1, a putative transcription factor in chickpea (Cicer arietinum L.), promotes stress tolerance. Plant Mol Biol. 2012; 79:479-93.

12. Bao $Y$, Song WM, Jin $Y L$, Jiang $C M$, Yang $Y$, Li B, Huang WJ, Liu $H$, Zhang $H X$ Characterization of Arabidopsis tubby-like proteins and redundant function of AtTLP3 and AtTLP9 in plant response to ABA and osmotic stress. Plant Mol Biol. 2014:86:471-83.

13. Chen Y, Dai W, Sun B, Zhao Y, Ma Q. Genome-wide identification and comparative analysis of the TUBBY-like protein gene family in maize. Genes Genom. 2016;38:25-36. 
14. Du F, Xu JN, Li D, Wang XY. The identification of novel and differentially expressed apple-tree genes under low-temperature stress using highthroughput Illumina sequencing. Mol Biol Rep. 2015;42:569-80.

15. Du F, Xu JN, Zhan CY, Yu ZB, Wang XY. An obesity-like gene MdTLP7 from apple (Malus $\times$ domestica) enhances abiotic stress tolerance. Biochem Biophys Res Commun. 2014;445:394-7.

16. Kiyosue T, Wada M. LKP1 (LOV kelch protein 1): a factor involved in the regulation of flowering time in arabidopsis. Plant J. 2000;23:807-15.

17. Mas P, Kim WY, Somers DE, Kay SA. Targeted degradation of TOC1 by ZTL modulates circadian function in Arabidopsis thaliana. Nature. 2003:426:567-70.

18. Somers DE, Kim WY, Geng R. The F-box protein ZEITLUPE confers dosagedependent control on the circadian clock, photomorphogenesis, and flowering time. Plant Cell. 2004;16:769-82.

19. Baudry A, Ito S, Song YH, Strait AA, Kiba T, Lu S, Henriques R, et al. F-box proteins FKF1 and LKP2 act in concert with ZEITLUPE to control Arabidopsis clock progression. Plant Cell. 2010;22:606-22.

20. Takase T, Nishiyama Y, Tanihigashi H, Ogura Y, Miyazaki Y, Yamada Y, Kiyosue T. LOV KELCH PROTEIN2 and ZEITLUPE repress Arabidopsis photoperiodic flowering under non-inductive conditions, dependent on FLAVIN-BINDING KELCH REPEAT F-BOX1. Plant J. 2011;67:608-21.

21. Mukhopadhyay S, Jackson PK. The tubby family proteins. Genome Biol. 2011;12:225

22. Mukhopadhyay S, Wen X, Chih B, Nelson CD, Lane WS, Scales SJ, et al. TULP3 bridges the IFT-A complex and membrane phosphoinositides to promote trafficking of $\mathrm{G}$ protein-coupled receptors into primary cilia. Genes Dev. 2010;24:2180-93.

23. Reitz MU, Bissue JK, Zocher K, Attard A, Huckelhoven R, Becker K, et al. The subcellular localization of tubby-like proteins and participation in stress signaling and root colonization by the mutualist Piriformospora indica. Plant Physiol. 2012;160:349-64.

24. Munnik T, Nielsen E. Green light for polyphosphoinositide signals in plants. Cur Opin Plant Biol. 2011;14:489-97.

25. Shinozaki K, Yamaguchi-Shinozaki K, Seki M. Regulatory network of gene expression in the drought and cold stress responses. Cur Opin Plant Biol. 2003;6:410-7.

26. Yamaguchi-Shinozaki K, Shinozaki K. Organization of cis-acting regulatory elements in osmotic- and cold-stress-responsive promoters. Trends Plant Sci. 2005;10:88-94

27. Vierstra RD. The ubiquitin-26S proteasome system at the nexus of plant biology. Nat Rev Mol Cell Biol. 2009;10:385-97.

28. Clough SJ, Bent AF. Floral dip: a simplified method for agrobacteriummediated transformation of Arabidopsis thaliana. Plant J. 1998;16:735-43.

29. Livak KJ, Schmittgen TD. Analysis of relative gene expression data using real-time quantitative PCR and the 2(-Delta Delta C(T)) method. Methods. 2001;25:402-8

30. Jia H, Hao L, Guo X, Liu S, Yan Y, Guo X. A Raf-like MAPKKK gene, GhRaf19, negatively regulates tolerance to drought and salt and positively regulates resistance to cold stress by modulating reactive oxygen species in cotton Plant Sci. 2016;252:267-81.

31. Arnold K, Bordoli L, Kopp J, Schwede T. The SWISS-MODEL workspace: a web-based environment for protein structure homology modelling. Bioinformatics. 2006;22:195-201.

32. Benkert P, Biasini M, Schwede T. Toward the estimation of the absolute quality of individual protein structure models. Bioinformatics. 2011;27:343-50.

33. Biasini M, Bienert S, Waterhouse A, Arnold K, Studer G, Schmidt T, et al. SWISS-MODEL: modelling protein tertiary and quaternary structure using evolutionary information. Nucleic Acids Res. 2014;42:W252-8.

Ready to submit your research? Choose BMC and benefit from:

- fast, convenient online submission

- thorough peer review by experienced researchers in your field

- rapid publication on acceptance

- support for research data, including large and complex data types

- gold Open Access which fosters wider collaboration and increased citations

- maximum visibility for your research: over $100 \mathrm{M}$ website views per year

At BMC, research is always in progress.

Learn more biomedcentral.com/submissions 